Draft VERsion November 5, 2018

Preprint typeset using ${ }^{A} \mathrm{~T}_{\mathrm{E}} \mathrm{X}$ style AASTeX6 v. 1.0

\title{
MULTI-FILTER TRANSIT OBSERVATIONS OF HAT-P-3b AND TrES-3b WITH MULTIPLE NORTHERN HEMISPHERE TELESCOPES
}

\begin{abstract}
D. Ricci ${ }^{1,2,3}$, P. V. SAdA ${ }^{4}$, S. NAvarro-Meza ${ }^{3}$, R. López-VAldivia ${ }^{5}$, R. Michel ${ }^{3}$, L. Fox Machado ${ }^{3}$, F. G. Ramón-Fox ${ }^{6}$, C. Ayala-Loera ${ }^{3,15}$, S. Brown Sevilla ${ }^{7}$, M. Reyes-Ruiz ${ }^{3}$, A. LA Camera ${ }^{8}$, C. Righi ${ }^{9,10}$

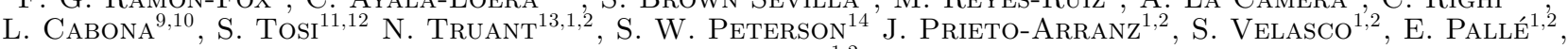
H. DEEG ${ }^{1,2}$
\end{abstract}

${ }^{1}$ Instituto de Astrofísica de Canarias, E-38205 La Laguna, Tenerife, Spain

2 Universidad de La Laguna, Departamento de Astrofísica, E-38206 La Laguna, Tenerife, Spain

${ }^{3}$ Observatorio Astronómico Nacional, Instituto de Astronomía - Universidad Nacional Autónoma de México, Ap. P. 877, Ensenada, BC 22860, México

${ }^{4}$ Departamento de Física y Matemáticas - Universidad de Monterrey, Avenida Ignacio Morones Prieto 4500 Pte., Jesús M. Garza, 66238 San Pedro Garza García, N.L., México

${ }^{5}$ Instituto Nacional de Astrofísica, Óptica y Electrónica, Luis Enrique Erro 1, Tonantzintla, Puebla 72840 México

6 SUPA, School of Physics and Astronomy, University of St Andrews, North Haugh, KY16 9SS, Scotland, UK

${ }^{7}$ Facultad de Ciencias Físico-Matemáticas, Benemérita Universidad Autónoma de Puebla, Av. San Claudio y 18 Sur, 72570 Puebla, México.

8 Dipartimento di Informatica, Bioingegneria, Robotica e Ingegneria dei Sistemi (DIBRIS), Università di Genova, Via Dodecaneso 35,16146 Genova, Italy

${ }^{9}$ Università degli Studi dell'Insubria, via Valleggio 11, 22100 Como, Italy

10 INAF-Osservatorio Astronomico di Brera, via Bianchi 46, 23807 Merate (LC), Italy

11 Dipartimento di Fisica - Università degli studi di Genova, Via Dodecaneso 33, 16146 Genova, Italy

12 Istituto Nazionale di Fisica Nucleare - Sezione di Genova, Via Dodecaneso 33, 16146 Genova, Italy

13 Dipartimento di Fisica - Università degli Studi di Trieste, Via Alfonso Valerio, 2, 34127 Trieste, Italy

14 Kitt Peak National Observatory, National Optical Astronomy Observatory, 950 N Cherry Ave., Tucson, AZ, 85719, USA

15 Observatório Nacional MCTIC, Rua Geral José Cristino 77, Rio de Janeiro, RJ, 20921-400, Brasil.

\begin{abstract}
We present a photometric follow-up of transiting exoplanets HAT-P-3b and TrES-3b, observed by using several optical and near-infrared filters, with four small-class telescopes $(D=36-152 \mathrm{~cm})$ in the Northern Hemisphere. Two of the facilities present their first scientific results. New 10 HAT-P-3b light curves and new 26 TrES-3b light curves are reduced and combined by filter in order to improve the quality of the photometry. Combined light curves fitting is carried out independently by using two different analysis packages, allowing the corroboration of the orbital and physical parameters in the literature. Results find no differences in the relative radius with the observing filter. In particular, we report for HAT-P-3b a first estimation of the planet-to-star radius $R_{p} / R_{*}=0.1112_{-0.0026}^{+0.0025}$ in the $B$ band which is coherent with values found in the $V R I z^{\prime} J H$ filters. Concerning TrES-3b, we derive a value for the orbital period of $P=1.3061862 \pm 0.0000001$ days which shows no linear variations over nine years of photometric observations.
\end{abstract}

Keywords: planets and satellites: fundamental parameters, (stars:) planetary systems

\section{INTRODUCTION}

The literature of exoplanetary transit observations started with the works of Charbonneau et al. (2000) and Henry et al. (2000) who separately confirmed radial velocity shifts of the star HD 209458 (Vogt et al. 1994; Baranne et al. 1996) due to the presence of its companion HD 209458 b. Since then, big efforts have been made in order to build up ground-based survey projects such as WASP (Pollacco et al. 2006), HAT
(Bakos et al. 2004) and its implementation HATSouth in the Southern Hemisphere (Penev et al. 2011; Bakos 2011), TrES (Alonso et al. 2007), XO (McCullough et al. 2005), KELT (Pepper et al. 2007) and the recent NGTS (Wheatley et al. 2013).

Among the current and forthcoming dedicated spacebased programs we mention Kepler (Borucki et al. 2010), PLATO (Rauer et al. 2014), and TESS (Ricker et al. 2010), as well as the European CHEOPS mis- 
Table 1. Main characteristics of observing facilities and their photometric instruments and altitude on the map on the right.

\begin{tabular}{|c|c|c|c|c|c|c|}
\hline & Telescope & & OAN-SPM & UDEM & IAC-TCS & OARPAF \\
\hline & Diameter & $\mathrm{cm}$ & 84 & 36 & 152 & 80 \\
\hline & Focal ratio & $f /$ & 15.0 & 10.0 & 13.8 & 8 \\
\hline & Latitude & $\mathrm{N}$ & $31^{\circ} 2^{\prime} 39^{\prime \prime}$ & $25^{\circ} 40^{\prime} 17.0^{\prime \prime}$ & $28^{\circ} 18^{\prime} 1.8^{\prime \prime}$ & $44^{\circ} 35^{\prime} 27.9^{\prime \prime}$ \\
\hline & Longitude & $\mathrm{W}$ & $115^{\circ} 27^{\prime} 49^{\prime \prime}$ & $100^{\circ} 18^{\prime} 31.0^{\prime \prime}$ & $16^{\circ} 30^{\prime} 39.2^{\prime \prime}$ & $350^{\circ} 47^{\prime} 47.0^{\prime \prime}$ \\
\hline & Instrument & & MEXMAN & SBIG STL-1301E & WIDE-FASTCAM & SBIG STL $11000 \mathrm{M}$ \\
\hline & CCD size & $\mathrm{px}$ & $2043 \times 4612$ & $1280 \times 1024$ & $1024 \times 1024$ & $2004 \times 1336$ \\
\hline 0 & Resolution & $\operatorname{arcsec} / \mathrm{px}$ & 0.25 & 1.0 & 0.50 & 0.29 \\
\hline & Field of View & $\operatorname{arcmin}$ & $8.4 \times 19.0$ & $21.3 \times 17.1$ & $8 \times 8$ & $10 \times 10$ \\
\hline & Gain & $e^{-} / \mathrm{ADU}$ & 1.8 & 2.3 & 0.10 & 0.8 \\
\hline 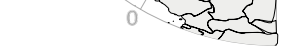 & Read Out Noise & $e^{-}$ & 4.8 & 16 & $2.7(\mathrm{~g} 1 \mathrm{~m} 200)$ & 12 \\
\hline
\end{tabular}

sion (Fortier et al. 2014), which will help to infer and characterize the exoplanetary population, and has the ambition to detect exomoons (Simon et al. 2015) and other interesting features. Such a huge amount of data can benefit from useful online databases such as ETD (Poddaný et al. 2010) ${ }^{1}$, and exoplanets.eu ${ }^{2}$ (Schneider et al. 2011) to help astronomers in programming their observations or defining best candidates for an independent follow-up.

In this framework, we have recently shown (Ricci et al. 2015) the adequacy of the San Pedro Mártir - Observatorio Astronómico Nacional (OAN-SPM) facilities, located in Northwest Mexico, as a valid resource in the Northern Hemisphere for the follow-up of exoplanetary transits, particularly that of Hot Jupiters. The survey, which started in 2014 (Ricci et al. 2014) and is still ongoing, has allowed, to date, the observation of a total of 30 transiting exoplanetary systems ${ }^{3}$, mainly with the $84 \mathrm{~cm}$ telescope, achieving good quality light curves in several filters, most of them through the Johnson's $V R I$ filters.

In this paper we focus on two objects: the Jupitersized HAT-P-3b and the grazing transiting planet TrES$3 \mathrm{~b}$, which we present in Sects. 2.1 and 2.2, respectively. Moreover, we will present data from other sites by using not only OAN-SPM survey data, but also light curves obtained from the following facilities: the Observatorio de la Universidad de Monterrey (UDEM) in the state of Nuevo Léon, Mexico; the Telescopio Carlos Sánchez (TCS) at the Observatorio del Teide (OT) in Tenerife, Spain, provided with WIDE-FASTCAM, a new concept of fast camera with a wide Field of View (FoV); and

\footnotetext{
1 See for example observations provided by Phil Evans and flagged with a quality of "2" and " 3 " http://var2. astro.cz/ETD/ etd.php?STARNAME=WASP-39\&PLANET $=b$, used to complement Ricci et al. (2015) data.

2 http://exoplanets.eu

3 http://www.astrosen.unam.mx/ indy/spm-transits/
}

the Osservatorio Astronomico Regionale Parco Antola, comune di Fascia (OARPAF) in northern Italy. All the telescopes and the relative instruments involved in our observations are described in Sect. 3.1. In particular, WIDE-FASTCAM is a new instrument, and OARPAF is a new facility, and we present their first scientific results. In Sects. 3.2 and 4 we focus on observations and data reduction of HAT-P-3b and TrES-3b. Conclusions are described in Sect. 5 .

\section{TARGETS}

\section{1. $H A T-P-3$}

In the framework of the HATNet survey, Torres et al. (2007) discovered HAT-P-3b, a Jupiter-size planet with a period of $2.899703 \pm 0.000054$ days, which was the smallest of the 18 transiting extrasolar planets known at that time, with a planet radius $R_{p}=0.890 \pm 0.046 R_{J}$, where $R_{J}$ stands for the radius of Jupiter. HAT-P3b immediately appeared to be metal-rich, with a content representing 75 Earth masses $M_{\oplus}$, (or, in Jupiter masses, $0.24 M_{J}$ ). Radial velocity measurements allowed authors to estimate a planetary mass of $M_{p}=$ $0.599 \pm 0.026 M_{J}$, and found a distance between planet and parent star of $0.03894 \pm 0.00070 \mathrm{AU}$, assuming no orbital eccentricity.

System parameters with an improved precision were then updated by Gibson et al. (2010) by fitting seven light curves obtained after performing aperture photometry on short-exposure (4-5s), wide-band filter (500700nm) Liverpool Telescope data. Particular attention was given to possible Earth-mass planets in the inner and outer 2 : 1 orbital resonance, but the lack of evidence of Transit Timing Variations (TTVs) excluded this hypothesis. Furthermore, Nascimbeni et al. (2011, 2012) refined the orbital parameters and ephemerides with a timing accuracy of $11 \mathrm{~s}$, demonstrating the potential of the observing and reduction strategy of the 
Table 2. Log of HAT-P-3b observations.

\begin{tabular}{|c|c|c|c|}
\hline Date (UT) & Filter & Exp.time & Observatory \\
\hline 2009-01-19 & $I c$ & $60 \mathrm{~s}$ & UDEM \\
\hline 2009-04-22 & Ic & $60 \mathrm{~s}$ & UDEM \\
\hline 2009-05-15 & $J H$ & $30 \mathrm{~s}$ & $\mathrm{KPNO}^{\mathrm{a}}$ \\
\hline 2009-05-15 & $z^{\prime}$ & $60 \mathrm{~s}$ & KPNO-VCT \\
\hline 2009-05-21 & $I c$ & $60 \mathrm{~s}$ & UDEM \\
\hline $2010-05-27$ & $J H$ & $20 \mathrm{~s}$ & $\mathrm{KPNO}^{\mathrm{a}}$ \\
\hline $2010-05-27$ & $B$ & $45 \mathrm{~s}$ & KPNO-VCT \\
\hline 2012-04-22 & $I c$ & $60 \mathrm{~s}$ & UDEM \\
\hline 2012-05-24 & $I c$ & $60 \mathrm{~s}$ & UDEM \\
\hline 2013-06-02 & $I c$ & $60 \mathrm{~s}$ & UDEM \\
\hline 2014-04-01 & $R$ & $120 \mathrm{~s}$ & OAN-SPM \\
\hline 2014-03-13 & $R$ & $120 \mathrm{~s}$ & OAN-SPM \\
\hline 2014-03-16 & $I$ & $120 \mathrm{~s}$ & OAN-SPM \\
\hline 2014-05-13 & $V$ & $90 \mathrm{~s}$ & OAN-SPM \\
\hline
\end{tabular}

TASTE $^{4}$ survey.

The anomalous radius of HAT-P-3b, smaller than a pure hydrogen-helium planet suggested the presence of a heavy-element core estimated to be $100 M_{\oplus}$. This was the subject of the investigation of Chan et al. (2011, 2012), who observed the target in the $i$ and $z^{\prime}$ bands, again finding no deviations in the timing of eclipses. Chan et al. (2011) found a period of $P=$ $2.8997360 \pm 0.0000020$ days, a planet-to-star radius ratio of $R_{p} / R_{*}=0.1063 \pm 0.0020$, an orbital inclination of $i=87.07 \pm 0.55^{\circ}$, and a scaled semi-major axis of $a / R_{*}=10.39 \pm 0.49$, adopting a circular orbit. An additional follow-up at Kitt Peak National Observatory was carried out by Sada et al. (2012), while Southworth (2012) conducted a homogeneous study of more than 30 extrasolar planets including HAT-P-3b, based on all available data sets in the literature. The author found a larger stellar radius $\left(0.947_{-0.027}^{+0.015} M_{J}\right)$ and pointed out that further photometric monitoring was needed to get rid of the discrepancy between the data sets of Torres et al. (2007); Gibson et al. (2010); Nascimbeni et al. (2011) and Chan et al. (2011). Finally, a space-based investigation of secondary eclipses in 3.6-4.5um spectral bands of the Spitzer Space Telescope was carried out by Todorov et al. (2013), finding inefficiencies in heat redistribution, but letting the scenario open about thermal inversion in the atmosphere. Moreover, Todorov et al. (2013) found the eccentricity of HAT-P-3b consistent with zero.

\footnotetext{
4 The Asiago Search for Transit timing variations of Exoplanets
}

Table 3. Log of TrES-3b observations.

\begin{tabular}{cccc}
\hline \hline Date (UT) & Filter & Exp.time & Observatory \\
\hline $2007-07-23$ & $I c$ & $120 \mathrm{~s}$ & UDEM \\
$2007-08-09$ & $I c$ & $120 \mathrm{~s}$ & UDEM \\
$2008-07-11$ & $I c$ & $60 \mathrm{~s}$ & UDEM \\
$2008-07-15$ & $V$ & $90 \mathrm{~s}$ & UDEM \\
$2008-10-04$ & $V$ & $90 \mathrm{~s}$ & UDEM \\
$2009-05-06$ & $z^{\prime}$ & $45 \mathrm{~s}$ & KPNO-VCT \\
$2009-05-10$ & $z^{\prime}$ & $120 \mathrm{~s}$ & KPNO-VCT \\
$2009-05-31$ & $I c$ & $120 \mathrm{~s}$ & UDEM \\
$2009-07-17$ & $z^{\prime}$ & $120 \mathrm{~s}$ & UDEM \\
$2010-04-04$ & $z^{\prime}$ & $40 \mathrm{~s}$ & StPr \\
$2010-04-25$ & $V$ & $90 \mathrm{~s}$ & UDEM \\
$2010-06-19$ & $V$ & $90 \mathrm{~s}$ & UDEM \\
$2010-07-19$ & $V$ & $90 \mathrm{~s}$ & UDEM \\
$2010-08-05$ & $I c$ & $90 \mathrm{~s}$ & UDEM \\
$2011-04-27$ & $I c$ & $90 \mathrm{~s}$ & UDEM \\
$2011-06-08$ & $R c$ & $60 \mathrm{~s}$ & UDEM \\
$2011-08-24$ & $R c$ & $60 \mathrm{~s}$ & UDEM \\
$2012-08-12$ & $R c$ & $60 \mathrm{~s}$ & UDEM \\
$2013-08-14$ & $R c$ & $60 \mathrm{~s}$ & UDEM \\
$2013-10-08$ & $V$ & $90 \mathrm{~s}$ & UDEM \\
$2014-06-13$ & $R c$ & $60 \mathrm{~s}$ & UDEM \\
$2015-07-17$ & $R$ & $120 \mathrm{~s}$ & TCS \\
$2015-08-03$ & $R$ & $120 \mathrm{~s}$ & OARPAF \\
$2015-08-22$ & $R$ & $120 \mathrm{~s}$ & OAN-SPM \\
$2016-04-09$ & $R$ & $120 \mathrm{~s}$ & OAN-SPM \\
$2016-04-13$ & $R$ & $120 \mathrm{~s}$ & OAN-SPM \\
binning & individual frames & of & 10s each.
\end{tabular}

\section{2. $\operatorname{Tr} E S-3 b$}

TrES-3b is a massive transiting Hot Jupiter discovered by O'Donovan et al. (2007) and confirmed by radial velocity measurements. Authors derived a period of $P=1.30619 \pm 0.00001$ days, a semi-major axis of $a=0.0226 \pm 0.0013 \mathrm{AU}$ and a near-grazing inclination value of $i=82.15 \pm 0.21^{\circ}$. The mass of the planet is estimated to be $M_{p}=1.92 \pm 0.23 M_{J}$ in front of a stellar mass of $M=0.9 \pm 0.15 M_{\odot}$, while the planet radius is $R_{p}=1.295 \pm 0.081 R_{J}$. de Mooij \& Snellen (2009) reported a temperature of the planet of $T=2040 \pm 185 \mathrm{~K}$ by measuring the thermal emission in the $K$ band using secondary eclipse observations which were carried out with the William Hershel Telescope, that complemented the space-based observations of the Spitzer Telescope.

The same value was found by de Mooij \& Snellen 
(2011). de Mooij \& Snellen (2009) also suggest that the planet is in a non-circular orbit. Measurements of the radius of the planet in the $K$ band do not significantly differ from the results obtained with optical observations.

Many follow-ups were carried out by several groups in optical and UV bands (Turner et al. 2011; Jones et al. 2012; Walker-LaFollette et al. 2012; Vaňko et al. 2012; Smith et al. 2012). In particular, Turner et al. (2013) did not detect any early ingress in UV as predicted by Vidotto et al. (2011), resulting in an abnormally small strength of the magnetic field. The observations in the near-infrared by de Mooij \& Snellen (2009), and the near UV by Turner et al. (2013), do not show differences in the value of the radius of the planet.

An attempt to calculate TTVs was made for the first time by Thakur et al. (2013), using 32 transits in the existing literature, and 5 new transits. Authors use dynamic models to suggest the presence of an additional outer planet close to the $1: 2$ resonance, with an estimated mass, in terms of Earth masses, $\approx 100 M_{\oplus}$. The result contradicts that found by Kundurthy et al. (2013), which observed an additional set of 11 transits in the framework of the Apache Point Survey of Transit Lightcurves of Exoplanets (APOSTLE), excluding the possibility of other planets and confirming the system's parameters but with reduced error bars. Finally, transit times of TrES-3b were updated by Maciejewski et al. (2013), whose results also support no TTVs.

\section{OBSERVATIONS}

\subsection{Facilities}

Observations presented in this paper were carried out by using four different telescopes located in the Northern Hemisphere. A summary of their characteristics is shown in Table 1.

- The first telescope is the OAN-SPM $84 \mathrm{~cm}$. It provides a set of instruments that can be mounted according to the observational needs, among which MEXman, a wide-field imager already successfully tested for the observation and characterization of exoplanets using the transit method (Ricci et al. 2015).

- The second telescope is the UDEM $36 \mathrm{~cm}$, a LX200GPS college telescope located close to the city of Monterrey, Mexico, and classed with a Minor Planet Center Code of 720 . It is available for student training and has been active for almost 10 years in the field of exoplanetary transit observations Ramón-Fox \& Sada (2013); Sada \& RamónFox (2016).
- The third telescope, the TCS, is a $152 \mathrm{~cm}$ equatorial Cassegrain telescope ${ }^{5}$ located at the OT and managed by the Astrophysics Institute of Canary Islands (IAC), Spain. This telescope is provided with different instruments. One of them, FAsTCAM, allows quasi-diffraction limited real-time observations using the Lucky Imaging technique, the implementation of the instrument being targeted to high temporal resolution up to tens of images per second (Oscoz et al. 2008). Despite these advantages, FASTCAM is limited (Murga et al. 2010) by its $20^{\prime \prime} \times 20^{\prime \prime}$ FoV.

For this reason, an implementation of the FASTCAM detector with a wider FoV was carried out: WidE-FASTCAM offers a $1024 \times 1024$ px EMCCD array and an optical design (Murga et al. 2014) to provide observers with an $\approx 8 \times 8^{\prime} \mathrm{FoV}$. We used WidE-FASTCAM for our observations, in order to test its small readout time and low electronic noise. Linearity tests on the camera on flat field images show that the instrument works in the linear regime between 1700 and 4000 counts (Velasco et al. 2016, 2017). During observations we took care to tune the exposure time accordingly.

- The fourth telescope is the alt-azimuthal ${ }^{6}$ OARPAF $80 \mathrm{~cm}$, located near Mt. Antola in Northern Italy, and whose scientific activity is managed by the Physics Department (DIFI) of the University of Genova, Italy. The telescope was designed by the Astelco company ${ }^{7}$ to foresee a double focal station: the first, provided with a field derotator, is dedicated to scientific observations, and currently equipped with an air-cooled SBIG STL $11000 \mathrm{M}$ camera and a standard $U B V R I$ Johnson filter wheel (Federici et al. 2012); the second is dedicated to ocular observations by amateurs.

Pointing and positioning of the secondary mirror are controlled using the proprietary AsteloS software, provided by the constructor, on a dedicated Linux machine, while the image data capture is managed by the MaxIm software. The time stamp is obtained via a Global Positioning System (GPS) device. The instrument was recently fullycharacterized with standard stars observations in all available filters for zero-point determination and extinction coefficient determination. Concern-

\footnotetext{
5 http://ww.iac.es/telescopes/pages/es/inicio/ telescopios/tcs.php

6 https://www.difi.unige.it/it/ricerca/altri-progetti/ osservatorio-monte-antola

7 http://www.astelco.com
} 
ing the CCD commissioning, deep tests allowed calculation of: gain, Read Out Noise, dark current, plate scale, and linearity regime (Righi 2016).

\subsection{Targets}

We carried out 4 observations of HAT-P-3b at OANSPM in nearly-full moon conditions, in 2014, over a period spanning five months using three filters: two with the $R$ filter, one with the $I$ filter and the final one with the $V$ filter. Except for the first $R$ observation, we were able to follow the target for roughly five hours. We also obtained 6 transits from UDEM taken with the $I c$ filter between 2009 and 2013. Moreover, we also aggregated 4 already published observations from Sada et al. (2012): two light curves from the Kitt Peak National Observatory (KPNO) $200 \mathrm{~cm}$ telescope observed with the $J H$ filter, one light curve from the KPNO Visitor Center Telescope (KPNO-VCT) $51 \mathrm{~cm}$ telescope with the $B$ filter and one curve from the same telescope with the Sloan $z^{\prime}$ filter. A log of HAT-P-3b observations is shown in Table 2 .

Concerning TrES-3b, we obtained three complete light curves in the $R$ band at OAN-SPM, and two in the $I$ band. Except for the first $I$ light curve, we used the defocused photometry method described in detail by Southworth et al. (2014) and in previous papers of the series. We also present 18 UDEM transits: six with the $V$ filter, five with the $R c$ filter, six in the $I c$ band and finally one in the $z^{\prime}$ band. Additional $z^{\prime}$ data come from KPNO (2 curves). An additional curve in the $z^{\prime}$ band comes from the KPNO-VCT operator Steven Peterson (StPr) using his private observatory, and located close to the KPNO facilities. TCS also observed the target one night by using WIDE-FASTCAM and short-exposure images of $1 \mathrm{~s}$ each, in the $R$ band. Finally, one observation with the $R$ filter was carried out at OARPAF. We carefully checked that the targets and reference stars were not relaying too close to hot or bad pixels or bad lines of devices, nor at the edge of their FoV. We also used a $2 \times 2$ binning for our observations in OAN-SPM, UDEM and OARPAF, windowing the devices in order to minimize the readout time. OAN-SPM, UDEM and OARPAF telescopes were also slightly defocused in order to spread the Point Spread Function over a large number of pixels, allowing longer exposure times, a shorter total readout time, and reducing errors due to pixel response or flat-field correction problems. A log of TrES-3b observations is shown in Table 3.

\section{DATA ANALYSIS}

Images of both targets were debiased and flat-field corrected. We used the aperture photometry technique to obtain light curves. In particular, we used the defot routine (Southworth et al. 2010) written in the IDL lan- guage, that we modified to work with FITS headers generated by the OAN-SPM, TCS and OARPAF telescopes, while UDEM data were reduced using independent custom IDL routines. OAN-SPM and OARPAF images were not aligned, as we decided to calculate and follow, for each image, the centroid of the target and that of the reference stars, by using a cross-correlation method that we re-implemented in defot. For the OARPAF data coming from an Alt-Az telescope, we set up a rotation tracking subroutine in order to correct for the residual rotation of the field due to eventual errors in the alignment between the optical axis, the derotator, and the CCD center.

Several field stars were tested in order to find a reference from which performing differential photometry, and for both HAT-P-3b and TrES-3b we chose a number between 2 and 8 non-saturated reference stars with count values as close as possible to those of the target, also taking into account sky conditions and availability.

A first-order trend in light curves was removed with the technique described by Ramón-Fox \& Sada (2013), in order to get rid of airmass effects. Timestamps of the light curve points were converted to the Dynamical Time-based system $\left(B J D_{\mathrm{TDB}}\right)$ applying the transformation given by Eastman et al. (2010).

Data were then combined by using the procedure described by Sada \& Ramón-Fox (2016). Using this procedure, we obtained a total of 7 combined curves for HATP-3b for each one of the following filters: $B$ (1 curve from KPNO-VCT), $V$ (1 curve from OAN-SPM), $R$ (2 curves from OAN-SPM), $I$ (1 curve from OAN-SPM), Ic ( 6 curves from UDEM), $z^{\prime}$ (1 curve from KPNO-VCT), $J H$ (2 curve from KPNO).

The same technique was applied to TrES-3b data, and we obtained a total of 6 combined curves in the different filters as follows: $V$ (6 curves from UDEM), $R$ (3 curves from OAN-SPM, 1 from TCS, 1 from OARPAF), $R c$ ( 5 curves from UDEM), $I$ (2 curves from OAN-SPM), Ic ( 6 curves from UDEM), $z^{\prime}$ (2 curves from KPNO, 1 from StPr, 1 from UDEM).

Light curves were fitted with EXOFAST (Eastman et al. 2012, 2013a). A value of the inclination $i$, of the semimajor axis in terms of the host star radius $a / R_{*}$, of the Mid-time $T_{m i d}$, and of the radius of the planet in terms of the host star radius $R_{p} / R_{*}$ were fitted for each combined curve. An independent light curve fitting was performed by using the IDL software Transit Analysis Package (TAP) implemented by Gazak et al. (2011). Both software use a Markov Chain Monte Carlo (MCMC) method to find best fit parameters for the Mandel \& Agol (2002) model.

Differing from EXOFAST, TAP allows fitting together, or separately, a set of parameters linking their value with a lock matrix. Thus it was possible to simultaneously fit 
HAT-P-3b combined light curves and fit

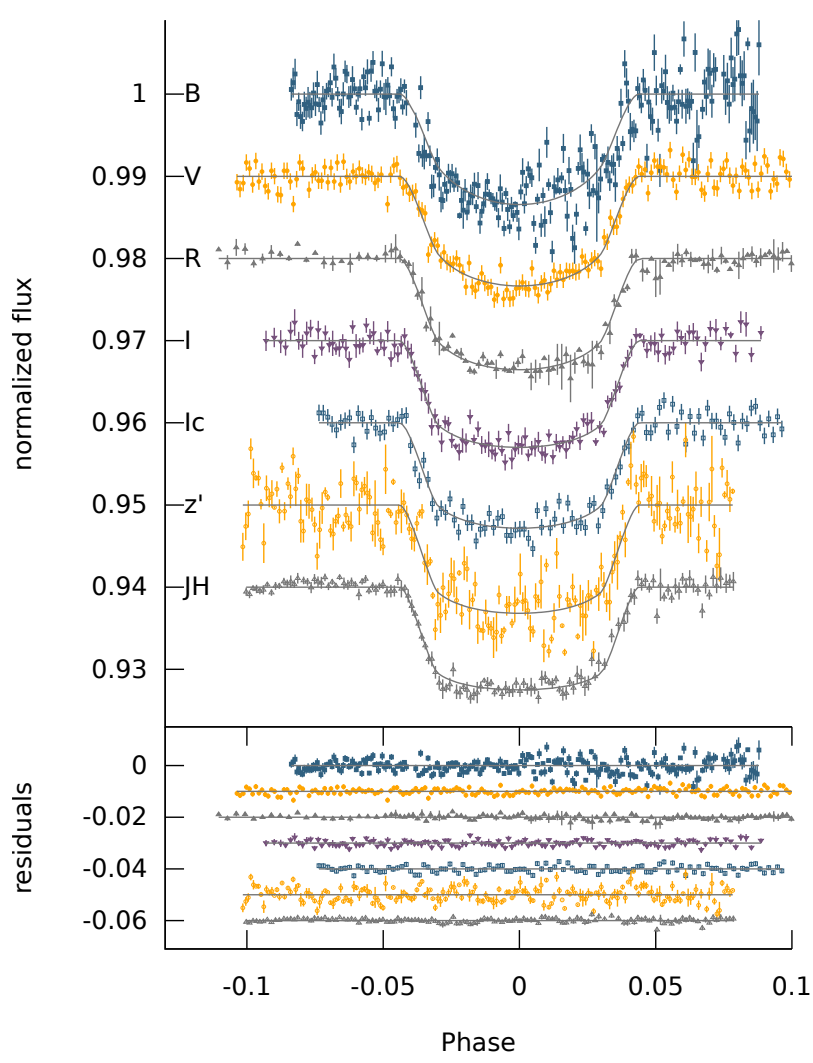

Figure 1. Combined light curves of HAT-P-3b and fit models. Models are slightly shifted in flux for better visualization. The lower panel shows residuals. See the online edition of the PASP for a color version of this figure.

a unique value of the inclination $i$ and of the scaled semimajor axis $a / R_{*}$ for all combined curves, a different Midtime $T_{m i d}$ value for each light curve, and a scaled planet radius $R_{p} / R_{*}$ value for each of the different observing filter.

\subsection{HAT-P-3b}

In fitting procedures we fixed the period: $P=$ 2.8997382 days (Sada et al. 2012), and we supposed a circular orbit (eccentricity $e=0$, argument of periastron $\omega=0$ ). Concerning limb darkening, for EXOFAST fitting we used interpolated values from Claret (2000), with the following parameters: $T_{*}=5224 \pm 69 \mathrm{~K}$, $\log g_{*}=4.58 \pm 0.03$, and $[\mathrm{Fe} / \mathrm{H}]_{*}=0.41 \pm 0.08$ (Torres et al. 2007, 2012); while for TAP fitting we used an online tool (Eastman et al. 2012, 2013b) which interpolates atmosphere models of Claret \& Bloemen (2011).

Fit results obtained with EXOFAST are shown in Table 4. In particular, weighted means obtained with EXOFAST are consistent with those of Nascimbeni et al. (2011): EXOFAST obtain $i=$ $86.73_{-0.11}^{+0.11}$ against $86.75_{-0.10}^{+0.10}$ of Nascimbeni et al. $(2011) ; a / R_{*}=10.13_{-0.11}^{+0.11}$ against $10.12_{-0.32}^{+0.32} ;$ and

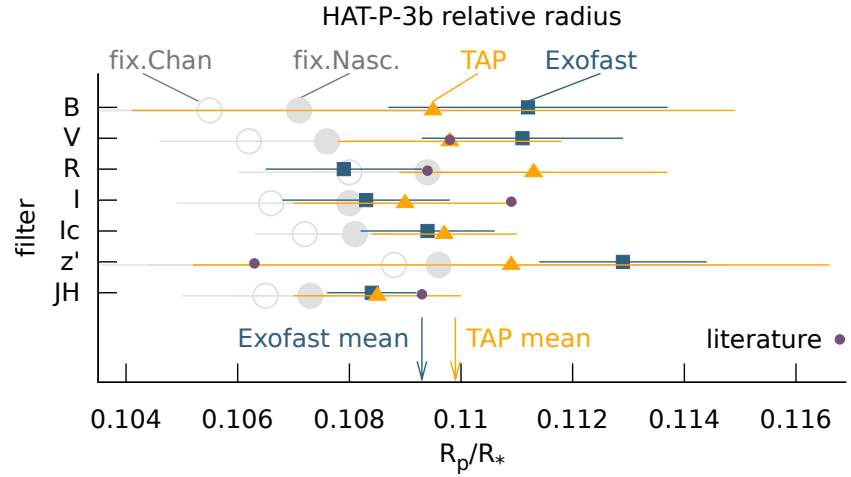

Figure 2. $\quad R_{p} / R_{*}$ values of HAT-P-3b for each observing filter resulting from EXOFAST and TAP fit procedures. Arrows show weighted means. Large light circles show TAP results obtained by fixing $i$ and $a / R_{*}$ with values provided by Chan et al. (2011) and Nascimbeni et al. (2011). Small bold circles show values provided in the literature: $V+R$ (Gibson et al. 2010), $R$ (Nascimbeni et al. 2011), $I$ (Torres et al. 2007), $z^{\prime}$ (Chan et al. 2011), and $J H$ (Sada et al. 2012) See the online edition of the PASP for a color version of this figure.

$R_{p} / R_{*}=0.1093_{-0.0005}^{+0.0005}$ against $0.1094_{-0.0011}^{+0.0011}$. TAP calculates a unique value for the first two parameters: the inclination $i=86.27_{-0.18}^{+0.28}$ and the scaled semi-major axis $a / R_{*}=9.65_{-0.18}^{+0.28}$, which are slightly different from ExOFAST and Nascimbeni et al. (2011) results, but in agreement within $3 \sigma$. A visual compairson between EXOFAST and TAP fit values of the scaled planet radius $R_{p} / R_{*}$ is shown in Fig. 2.

Fig. 2 also show additional TAP fit solutions obtained by fixing $i$ and $a / R_{*}$ found by Chan et al. (2011) and Nascimbeni et al. (2011), and fitting only a separate value of $R_{p} / R_{*}$ for each observing filter. The figure gives evidence of the fact that we find no significant radius variation with the observing filter. In Fig. 3 calculated Mid-times are shown.

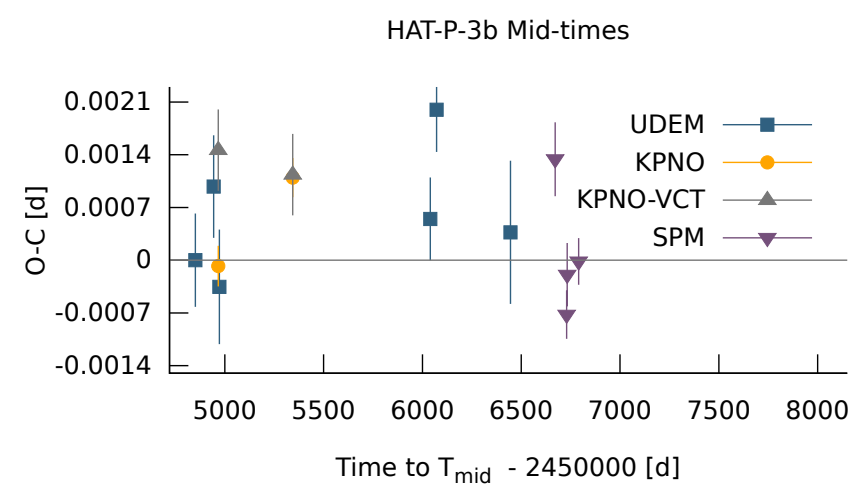

Figure 3. Observed minus Calculated Mid-times values for HAT-P-3b observations presented in this work. See the online edition of the PASP for a color version of this figure. 


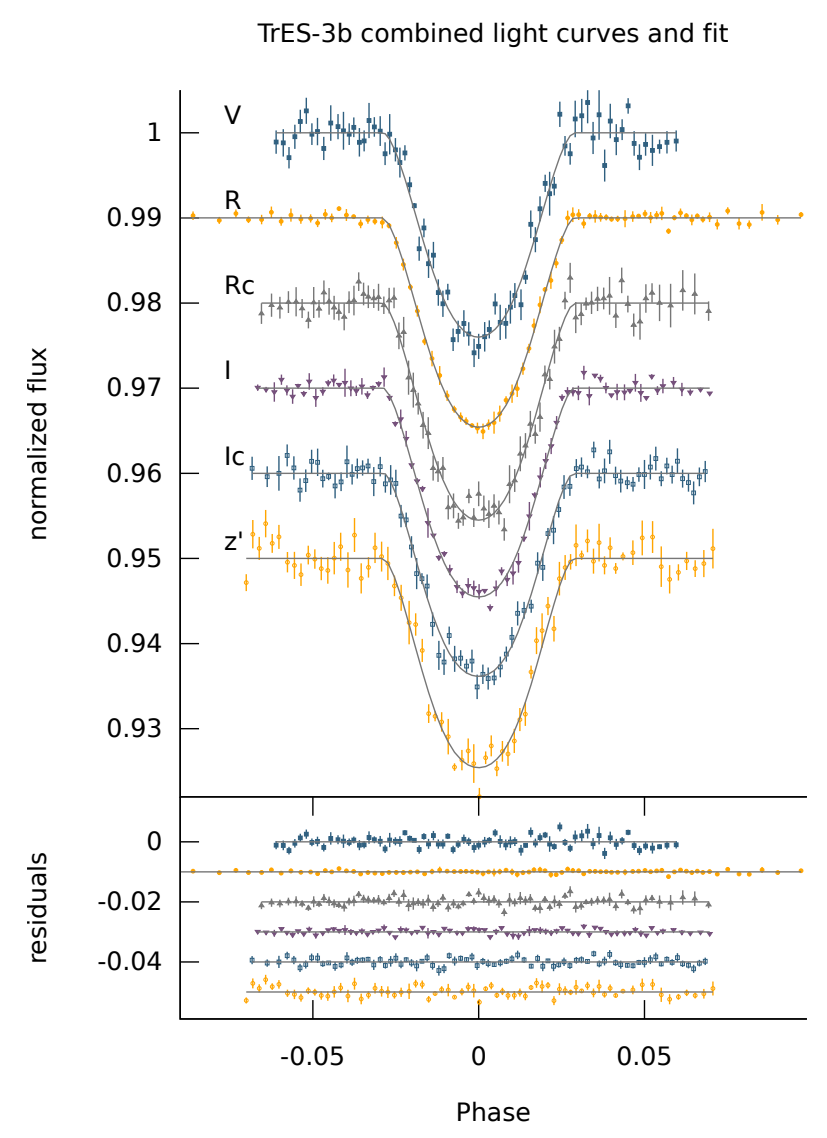

Figure 4. Light curves of TrES-3b and fit. The lower panel shows residuals. See the online edition of the PASP for a color version of this figure.

\section{2. $\operatorname{Tr} E S-3 b$}

We used the same technique applied for HAT-P-3b fitting for what concerns TrES-3b limb darkening, with following parameters: $\log \left(g_{*}\right)=4.571 \pm 0.006$ (Southworth 2011), $T_{*}=5650 \pm 75 \mathrm{~K}$, and $[\mathrm{Fe} / \mathrm{H}]_{*}=-0.19 \pm 0.08$ (Sozzetti et al. 2009).

EXOFAST fit results of TrES-3b are shown in Table. 5.

While performing the TAP fit, we fitted a unique value of $i$ and $a / R_{*}$ for all combined curves, and we fitted separately a value of $R_{p} / R_{*}$ for each considered filter. TAP results show good agreement with the EXOFAST weighted averages of $i$ and $a / R_{*}: i=81.70_{-0.22}^{+0.17}$ of TAP against $81.63_{-0.23}^{+0.20}$ of EXOFAST, and $a / R_{*}=5.885_{-0.080}^{+0.068}$ of TAP against $5.903 \pm 0.062$ of EXOFAST. Results are slightly lower than, but in agreement with, the values provided by Kundurthy et al. (2013): $i=81.86_{-0.26}^{+0.08}$ and $a / R_{*}=5.91_{-0.05}^{+0.04}$. Concerning $R_{p} / R_{*}$, we have differences between the two fit procedures which are within $-2 \sigma$ (for the $V$ filter) and $+1 \sigma$ (for the $I$ filter). Comparisons of $R_{p} / R_{*}$ between different filters show a maximum absolute variation of $2.9 \sigma$ between $R$ and $V$ curves in EXOFAST results, which are not confirmed by TAP results $(0.04 \sigma)$. We then assume no $R_{p} / R_{*}$ variations with the observing filters, and we consider average

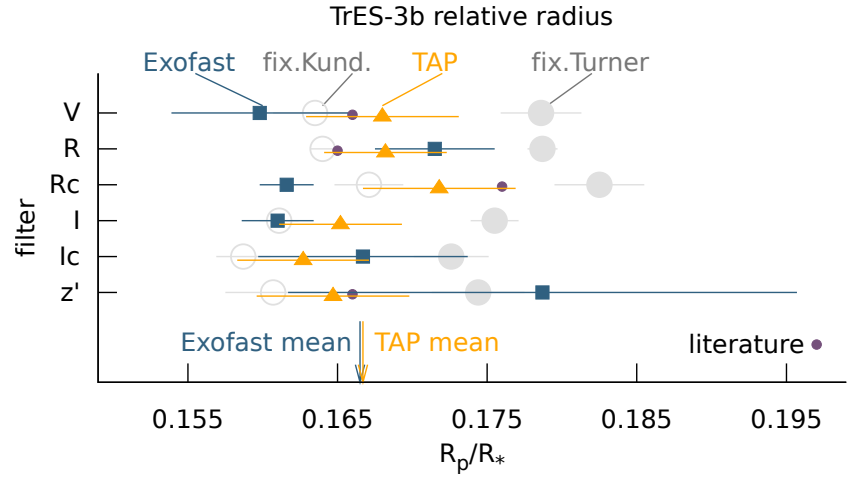

Figure 5. $\quad R_{p} / R_{*}$ values of TrES-3b for each observing filter resulting from EXOFAST and TAP fit procedures. Arrows show weighted means. Large light circles show TAP results obtained by fixing $i$ and $a / R_{*}$ with values provided by Kundurthy et al. (2013) and (Turner et al. 2013). Small bold circles show values provided in the literature: $V$ and $R c$ (Turner et al. 2013), $R$ (Kundurthy et al. 2013), and $z^{\prime}$ (O'Donovan et al. 2007). See the online edition of the PASP for a color version of this figure.

results finding good agreement between fit procedures: $R_{p} / R_{*}=0.1665_{-0.0050}^{+0.0081}$ for EXOFAST and $0.1667_{-0.0035}^{+0.0047}$ for TAP, against the value of $0.1649 \pm 0.0015$ provided by Kundurthy et al. (2013) in the $r^{\prime}$ band (see Fig. 5).

Fig. 5 also show additional TAP fit solutions obtained by fixing $i$ and $a / R_{*}$ found by Kundurthy et al. (2013) and (Turner et al. 2013), and fitting only a separate value of $R_{p} / R_{*}$ for each observing filter.

Mid-times fit linearly a period of $P=1.3061862 \pm$ 0.0000001 days (see Fig. 6). Scatter of calculated values of $T_{\text {mid }}$ corresponds to variations of $\approx 4$ minutes peakto-valley, mostly ruled by UDEM data, which is reduced to $\approx 1$ minute peak-to-valley while considering OANSPM, OARPAF and TCS data. Calculated Mid-times show no significant linear variation of the period with the time, and our sampling does not allow us to search for periodic variations in the $1: 2$ resonance as in the work of Thakur et al. (2013).

\section{TrES-3b Mid-times}

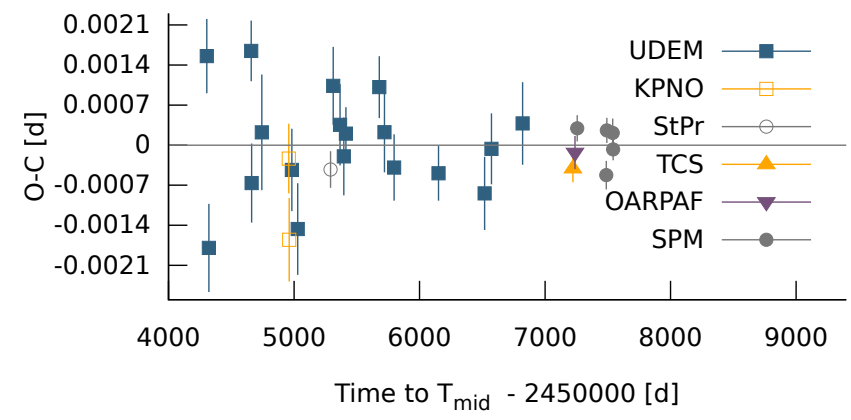

Figure 6. Observed minus Calculated Mid-times values for TrES-3b observations presented in this work. Data are consistent with a $P=1.3061862 \pm 0.0000001$. See the online edition of the PASP for a color version of this figure. 
Table 4. EXOFAST fit results for HAT-P-3b.

\begin{tabular}{ccccc}
\hline \hline & obs. & $i$ & $a / R_{*}$ & $R_{p} / R_{*}$ \\
\hline$B$ & KPNO-VCT & $86.78_{-0.27}^{+0.29}$ & $10.17_{-0.34}^{+0.37}$ & $0.1112_{-0.0026}^{+0.0025}$ \\
$V$ & OAN-SPM & $86.57_{-0.26}^{+0.27}$ & $10.19_{-0.32}^{+0.34}$ & $0.1111_{-0.0018}^{+0.0018}$ \\
$R$ & OAN-SPM & $86.83_{-0.39}^{+0.36}$ & $10.12_{-0.36}^{+0.34}$ & $0.1079_{-0.0012}^{+0.0014}$ \\
$I$ & OAN-SPM & $86.71_{-0.27}^{+0.29}$ & $10.18_{-0.31}^{+0.32}$ & $0.1083_{-0.0015}^{+0.0015}$ \\
$I c$ & UDEM & $86.74_{-0.29}^{+0.31}$ & $10.18_{-0.30}^{+0.32}$ & $0.1094_{-0.0012}^{+0.0012}$ \\
$z^{\prime}$ & KPNO-VCT & $88.96_{-0.90}^{+0.71}$ & $10.04_{-0.21}^{+0.16}$ & $0.1129_{-0.0015}^{+0.0015}$ \\
$J H$ & KPNO & $86.60_{-0.24}^{+0.25}$ & $10.16_{-0.28}^{+0.29}$ & $0.1084_{-0.0008}^{+0.000}$ \\
\hline
\end{tabular}

Table 5. EXOFAST fit results for TrES-3b.

\begin{tabular}{ccccc}
\hline \hline & obs. & $i$ & $a / R_{*}$ & $R_{p} / R_{*}$ \\
\hline$V$ & UDEM & $81.34_{-0.25}^{+0.23}$ & $5.903_{-0.066}^{+0.066}$ & $0.1598_{-0.0045}^{+0.0059}$ \\
$R$ & $\mathrm{a}$ & $81.80_{-0.17}^{+0.15}$ & $5.899_{-0.056}^{+0.054}$ & $0.1715_{-0.0030}^{+0.0040}$ \\
$R c$ & UDEM & $81.59_{-0.14}^{+0.15}$ & $5.913_{-0.059}^{+0.060}$ & $0.1616_{-0.0019}^{+0.0018}$ \\
$I$ & OAN-SPM & $81.77_{-0.16}^{+0.14}$ & $5.895_{-0.061}^{+0.061}$ & $0.1610_{-0.0015}^{+0.0024}$ \\
$I c$ & UDEM & $81.83_{-0.22}^{+0.19}$ & $5.902_{-0.064}^{+0.063}$ & $0.1667_{-0.0050}^{+0.0070}$ \\
$z^{\prime}$ & $\mathrm{b}$ & $81.46_{-0.37}^{+0.28}$ & $5.906_{-0.066}^{+0.067}$ & $0.1787_{-0.0095}^{+0.0170}$ \\
\hline \multicolumn{5}{c}{$a_{\text {Combining OAN-SPM, OARPAF, and TCS data. }}$ O Combining UDEM, KPNO, and StPr data. }
\end{tabular}

\section{CONCLUSIONS}

We obtained ten new exoplanetary transit observations of HAT-P-3b in the $B V R I z^{\prime} J H$ bands, and twenty-six new observations of TrES-3b in the $V R I z^{\prime}$ bands, which confirmed the potential adequacy of smallsize telescopes $(36-152 \mathrm{~cm})$ for this research topic. In particular, the new OARPAF observatory and the new instrument WIDE-FASTCAM at TCS can provide reliable photometric observations in the framework of ground-based exoplanetary transit follow-ups.

The simultaneous fit of light curves, carried out in multiple observing bands by several telescopes, allowed us to achieve orbital and physical parameters which corroborate results of Nascimbeni et al. (2011) for what concerns HAT-P-3b, and of Kundurthy et al. (2013) for what concerns TrES-3b.

We also report specific values of $R_{p} / R_{*}$ in multiple optical and near-infrared bands, and a first estimation of this parameter in the $B$ band for HAT-P-3b which is coherent with our values provided by using other filters.

We find that observing filters do not significantly influence the determination of the relative radius of HAT-P$3 \mathrm{~b}$ and TrES-3b. This result confirms the relative radius of HAT-P-3b estimated by near-infrared band observations (de Mooij \& Snellen 2009), and the relative radius of TrES-3b estimated by UV observations (Turner et al. 2013) in the literature.

Mid-times of TrES-3b fit a $P=1.3061862 \pm 0.0000001$ days, finding no significant linear variations of the period over $\approx 9$ years of photometric observations. Further observations are planned in order to discriminate eventual $R_{p} / R_{*}$ variations with the observing filter and to extend $T_{\text {mid }}$ information in order to estimate TTVs.

The authors acknowledge the following financial support: DR from the Spanish Ministry of Economy and Competitiveness (MINECO) under the 2011 Severo Ochoa Program MINECO SEV-2011-0187; LFM and RM from the UNAM under grant PAPIIT IN 105115; DR and FGRF from the CONACYT scholarship for postgraduate studies in Mexico. Research carried out thanks to the support of UNAM-DGAPA-PAPIIT project IN115413. SN thanks H. Navarro-Meza for assistance during OAN-SPM observations in 2016/06. DR thanks Claudia Molina and Katie Marley for language editing, and the anonymous referee for remarks. We also acknowledge the OAN staff for daily support. The $1.5 \mathrm{~m}$ Carlos Sánchez Telescope is operated on the island of Tenerife by the Instituto de Astrofísica de Canarias in the Spanish Observatorio del Teide.

Facilities:

Facility: OAN-SPM 84cm (MEXMAN), UDEM $80 \mathrm{~cm}$, TCS $152 \mathrm{~cm}$ (WIDE-FASTCAM), OARPAF $80 \mathrm{~cm}$

\section{REFERENCES}

Alonso, R., Brown, T. M., Charbonneau, D., et al. 2007, in Astronomical Society of the Pacific Conference Series, Vol. 366, Transiting Extrapolar Planets Workshop, ed. C. Afonso, D. Weldrake, \& T. Henning, 13

Bakos, G. 2011, in Bulletin of the American Astronomical Society, Vol. 43, American Astronomical Society Meeting Abstracts \#218, 103.04

Bakos, G., Noyes, R. W., Kovács, G., et al. 2004, PASP, 116, 266 Baranne, A., Queloz, D., Mayor, M., et al. 1996, A\&AS, 119, 373 Borucki, W. J., Koch, D., Basri, G., et al. 2010, Science, 327, 977 Chan, T., Ingemyr, M., Winn, J. N., et al. 2011, AJ, 141, 179 —. 2012, AJ, 144, 90

Charbonneau, D., Brown, T. M., Latham, D. W., \& Mayor, M. 2000, ApJL, 529, L45
Claret, A. 2000, A\&A, 363, 1081

Claret, A., \& Bloemen, S. 2011, A\&A, 529, A75

de Mooij, E., \& Snellen, I. 2011, in Astronomical Society of the Pacific Conference Series, Vol. 450, Molecules in the

Atmospheres of Extrasolar Planets, ed. J. P. Beaulieu,

S. Dieters, \& G. Tinetti, 59

de Mooij, E. J. W., \& Snellen, I. A. G. 2009, A\&A, 493, L35

Eastman, J., Gaudi, B. S., \& Agol, E. 2012, EXOFAST: Fast

transit and/or RV fitter for single exoplanet, Astrophysics

Source Code Library, , , ascl:1207.001

-. 2013a, PASP, 125, 83

—. 2013b, PASP, 125, 83

Eastman, J., Siverd, R., \& Gaudi, B. S. 2010, PASP, 122, 935 
Federici, A., Arduino, P., Riva, A., \& Zerbi, F. M. 2012, in Astronomical Society of India Conference Series, Vol. 7, 7

Fortier, A., Beck, T., Benz, W., et al. 2014, in Society of Photo-Optical Instrumentation Engineers (SPIE) Conference Series, Vol. 9143, Society of Photo-Optical Instrumentation Engineers (SPIE) Conference Series, 2

Gazak, J. Z., Johnson, J. A., Tonry, J., et al. 2011, Transit Analysis Package (TAP and autoKep): IDL Graphical User Interfaces for Extrasolar Planet Transit Photometry, Astrophysics Source Code Library, , , ascl:1106.014

Gibson, N. P., Pollacco, D. L., Barros, S., et al. 2010, MNRAS, 401, 1917

Henry, G. W., Marcy, G. W., Butler, R. P., \& Vogt, S. S. 2000, ApJL, 529, L41

Jones, M. L., Adams, E. R., \& Carter, J. A. 2012, in American Astronomical Society Meeting Abstracts, Vol. 219, American Astronomical Society Meeting Abstracts \#219, \#339.01

Kundurthy, P., Becker, A. C., Agol, E., Barnes, R., \& Williams, B. 2013, ApJ, 764, 8

Maciejewski, G., Puchalski, D., Saral, G., et al. 2013, Information Bulletin on Variable Stars, 6082, 1

Mandel, K., \& Agol, E. 2002, ApJL, 580, L171

McCullough, P. R., Stys, J. E., Valenti, J. A., et al. 2005, PASP, 117,783

Murga, G., Oscoz, A., López, R., et al. 2014, in Society of Photo-Optical Instrumentation Engineers (SPIE) Conference Series, Vol. 9147, Society of Photo-Optical Instrumentation Engineers (SPIE) Conference Series, 6

Murga, G., Sanquirce, R., Campo, R., et al. 2010, in Society of Photo-Optical Instrumentation Engineers (SPIE) Conference Series, Vol. 7735, Society of Photo-Optical Instrumentation Engineers (SPIE) Conference Series, 3

Nascimbeni, V., Piotto, G., Bedin, L. R., \& Damasso, M. 2011, A\&A, 527, A85

—. 2012, Memorie della Societa Astronomica Italiana Supplementi, 19, 105

O’Donovan, F. T., Charbonneau, D., Bakos, G. Á., et al. 2007, ApJL, 663, L37

Oscoz, A., Rebolo, R., López, R., et al. 2008, in Society of Photo-Optical Instrumentation Engineers (SPIE) Conference Series, Vol. 7014, Society of Photo-Optical Instrumentation Engineers (SPIE) Conference Series, 47

Penev, K., Csubry, Z., \& Bakos, G. 2011, in Bulletin of the American Astronomical Society, Vol. 43, American Astronomical Society Meeting Abstracts \#217, 253.01

Pepper, J., Pogge, R. W., DePoy, D. L., et al. 2007, PASP, 119, 923

Poddaný, S., Brát, L., \& Pejcha, O. 2010, NewA, 15, 297

Pollacco, D. L., Skillen, I., Collier Cameron, A., et al. 2006, PASP, 118, 1407

Ramón-Fox, F. G., \& Sada, P. V. 2013, RMxAA, 49, 71

Rauer, H., Catala, C., Aerts, C., et al. 2014, Experimental Astronomy, 38, 249

Ricci, D., Rámon Fox, G., Ayala, C., et al. 2014, in AAS/Division for Planetary Sciences Meeting Abstracts, Vol. 46, AAS/Division for Planetary Sciences Meeting Abstracts, 210.04

Ricci, D., Ramón-Fox, F. G., Ayala-Loera, C., et al. 2015, PASP, 127, 143

Ricker, G. R., Latham, D. W., Vanderspek, R. K., et al. 2010, in Bulletin of the American Astronomical Society, Vol. 42, American Astronomical Society Meeting Abstracts \#215, \#450.06
Righi, C. 2016, Nuovo Cimento C Geophysics Space Physics C, 39, 284

Sada, P. V., \& Ramón-Fox, F. G. 2016, PASP, 128, 024402

Sada, P. V., Deming, D., Jennings, D. E., et al. 2012, PASP, 124, 212

Schneider, J., Dedieu, C., Le Sidaner, P., Savalle, R., \& Zolotukhin, I. 2011, A\&A, 532, A79

Simon, A. E., Szabó, G. M., Kiss, L. L., Fortier, A., \& Benz, W. 2015, ArXiv e-prints, arXiv:1508.00321

Smith, C.-T. W., Smart, B., Turner, J. D., et al. 2012, in American Astronomical Society Meeting Abstracts, Vol. 220, American Astronomical Society Meeting Abstracts \#220, \#129.04

Southworth, J. 2011, MNRAS, 417, 2166

-. 2012, MNRAS, 426, 1291

Southworth, J., Mancini, L., Novati, S. C., et al. 2010, MNRAS, 408,1680

Southworth, J., Hinse, T. C., Burgdorf, M., et al. 2014, MNRAS, 444,776

Sozzetti, A., Torres, G., Charbonneau, D., et al. 2009, ApJ, 691, 1145

Thakur, P., Jiang, I.-G., Yeh, L.-C., et al. 2013, in Astronomical Society of India Conference Series, Vol. 9, Astronomical Society of India Conference Series, 78

Todorov, K. O., Deming, D., Knutson, H. A., et al. 2013, ApJ, 770,102

Torres, G., Fischer, D. A., Sozzetti, A., et al. 2012, ApJ, 757, 161

Torres, G., Bakos, G. Á., Kovács, G., et al. 2007, ApJL, 666, L121

Turner, J., Hardegree-Ullman, K., Smart, B., et al. 2011, in American Astronomical Society Meeting Abstracts \#218, \#128.11

Turner, J. D., Smart, B. M., Hardegree-Ullman, K. K., et al. 2013, MNRAS, 428, 678

Vaňko, M., Jakubík, M., Krejčová, T., et al. 2012, in IAU Symposium, Vol. 282, IAU Symposium, ed. M. T. Richards \& I. Hubeny, 135-136

Velasco, S., Etxegarai, U., Oscoz, A., et al. 2016, in Proc. SPIE, Vol. 9908, Society of Photo-Optical Instrumentation Engineers (SPIE) Conference Series, $99082 \mathrm{O}$

Velasco, S., Oscoz, A., López, R. L., et al. 2017, in Highlights on Spanish Astrophysics IX, Proceedings of the XII Scientific Meeting of the Spanish Astronomical Society held on July 18-22, 2016, in Bilbao, Spain, ISBN 978-84-606-8760-3. S. Arribas, A. Alonso-Herrero, F. Figueras, C.

Hernández-Monteagudo, A. Sánchez-Lavega, S. Pérez-Hoyos (eds.), 2017 , p. 707-707, ed. S. Arribas, A. Alonso-Herrero, F. Figueras, C. Hernández-Monteagudo, A. Sánchez-Lavega, \& S. Pérez-Hoyos, 707-707

Vidotto, A. A., Jardine, M., \& Helling, C. 2011, MNRAS, 414, 1573

Vogt, S. S., Allen, S. L., Bigelow, B. C., et al. 1994, in Society of Photo-Optical Instrumentation Engineers (SPIE) Conference Series, Vol. 2198, Instrumentation in Astronomy VIII, ed. D. L. Crawford \& E. R. Craine, 362

Walker-LaFollette, A., Turner, J. D., Hardegree-Ullman, K. K., et al. 2012, in American Astronomical Society Meeting Abstracts, Vol. 219, American Astronomical Society Meeting Abstracts \#219, \#339.09

Wheatley, P. J., Pollacco, D. L., Queloz, D., et al. 2013, in European Physical Journal Web of Conferences, Vol. 47, European Physical Journal Web of Conferences, 13002 